Conclusion. Average T2 differs significantly between lipomatous masses of variable differentiation score in a limited sample of benign and malignant lipomatous tumors. $2^{\prime}$ is larger for lipomas and well differentiated liposarcomas compared to intermediate or poorly differentiated liposarcomas suggesting lower oxygen supply/consumption of the lesion.

https://doi.org/10.1016/j.ejmp.2018.06.094

[OA023] Multiparametric MRI measurements of renal perfusion and oxygenation

Bryan Haddock ${ }^{*}$, Henrik Larsson, Ulrik Andersen

Rigshospitalet, Dep. of Clinical Physiology, Nuclear Medicine E Pet, Rigshospitalet, Copenhagen University Hospital, Copenhagen, Denmark * Corresponding author.

Purpose. Renal medullary flow abnormalities have been implicated in the pathogenesis of primary and secondary hypertension. Still, due to the difficulties involved, combined measurements of medullary perfusion and oxygenation have only recently been reported. Using MRI, we have measured changes in renal flow, perfusion and oxygenation in healthy humans during activation of the sympathetic nerve system and after injection of a diuretic.

Methods. We measured changes in renal perfusion and blood oxygenation in 10 healthy subjects during handgrip exercise (HGE) and after furosemide injections on two occasions in a 3 Tesla MRscanner. For the two interventions, renal arterial flow (RAF) data were acquired using phase contrast (PC-MRI), perfusion using arterial spin labeling (ASL) and changes in blood oxygenation were monitored using both $\mathrm{T}_{2}^{*}$ measurements and blood oxygenation dependent (BOLD) imaging.

Results. HGE induced a decrease of RAF by $17.4 \pm 7 \%$, and an increase of oxygenation with a $\Delta \mathrm{T}_{2}^{*}$ of $22 \pm 9 \%$ in the renal medulla despite a significant drop in medullary perfusion $(-12 \pm 5 \%)$. In the renal cortex on the other hand, both perfusion and oxygenation decreased significantly. After furosemide injection, mean $\mathrm{T}_{2}^{*}$ values in the renal medulla increased by $45 \%$ ( $\mathrm{p}<0.01$ ) while other parameters, including medullary perfusion, did not change significantly. Coefficients of variation (CV) between baseline measures between the two scanning days were $6.6 \%$ for renal artery flow. $\mathrm{T}_{2}^{*}$ and ASL perfusion in renal cortex had CV values of $4.2 \%, 5.1 \%$, and $6.4 \%$ and $11.2 \%$ respectively in the medulla.

Conclusion. Measurements of RAF, BOLD and perfusion in renal cortex and medulla were acquired for the first time in healthy subjects during hand-grip testing and furosemide injection. Measurements from both interventions reveal that while changes in RAF, cortical perfusion and cortical oxygenation were synonymous, medullary oxygenation increased independently of perfusion after a diuretic injection and contrary to decreased perfusion during HGE induced sympathetic activation. These techniques may be useful in pathophysiologic studies of patients with hypertension or renal artery stenosis, and a better understanding of the mechanisms regulating medullary oxygenation.

https://doi.org/10.1016/j.ejmp.2018.06.095
1024

Abstract not available.

\section{[OA025] Response of ionization chambers in the presence of magnetic fields}

Mohamad ALissa ${ }^{\mathrm{a},{ }^{*}}$, Damian Czarnecki ${ }^{\mathrm{b}}$, Philip Von Voigts Rhetz ${ }^{\mathrm{a}}$, Klemens Zink ${ }^{\text {a }}$

${ }^{\text {a }}$ Technische Hochschule Mittelhessen, Department of Radiotherapy and Radiooncology, University Medical Center Giessen-Marburg, Giessen, Germany, Institute of Medical Physics and Radiation Protection, Technische Hochschule Mittelhessen, Giessen, Germany

${ }^{\mathrm{b}}$ Technische Hochschule Mittelhessen, Institute of Medical Physics and Radiation Protection, Technische Hochschule Mittelhessen, Giessen, Germany

* Corresponding author.

Purpose. The next generation of medical electron linear accelerators will integrate Magnetic Resonance Tomography (MRI). Therefore it will be possible to take direct images of the moving tumor during radiotherapy treatment. However serious consideration must be given to the strong magnetic field, which has an impact on the trajectories of the produced secondary electrons, because of the Lorentz force. This force affects both the dose distribution in water and the dose response of used detectors. For an accurate patient dosimetry these effects must be taken into account.

Methods. Monte Carlo methods correctly describe the radiation transport in different media, even in presence of magnetic fields and are therefore the gold standard for the evaluation of the impact of magnetic fields on clinical dosimetry. In this present study, the beam quality correction factors $\mathrm{Kq}$ and the relative response in magnetic fields of different ion chambers (PTW31013, PTW-31021, EXRADIN-1ASL, NE2571) were investigated with Monte Carlo simulations, using the code EGSnrc. The chambers were modelled in detail according to the information given by the manufacturer and placed in a water phantom $(30 \times 30 \times$ $30 \mathrm{~cm}^{3}$ ). The chambers were irradiated under reference conditions, following the recommendations of present dosimetry protocols, such as IAEA TRS-398: i.e. the field size at the phantom surface was $10 \times 10 \mathrm{~cm}^{2}$; the focus-surface-distance $100 \mathrm{~cm}$ and the depth of the chamber's reference point was $10 \mathrm{~cm}$. The source of the photons were several spectra of clinical medical accelerators applying nominal energies between 4 and $24 \mathrm{MV}-\mathrm{X}$. The magnetic field was applied in different directions relative to the beam axis (z-direction) and the chamber's symmetry axis and was varied between 0 and $3 \mathrm{~T}$.

Results. All chambers successfully passed the Fano test, the response of all chambers varied up to about $8 \%$ depending on the magnetic field strength and the field directions.

Conclusions. The response of the chambers with the magnetic fields, has a complex dependence on chamber radius, magnetic field strength, the orientation between radiation beams, chamber axis and magnetic field, and lastly the energy spectrum. Those variables consequently played a major role in the increase or decrease of correction factor, Kq.

https://doi.org/10.1016/j.ejmp.2018.06.097 